\title{
ECOLOGIA DE Tropidurus torquatus (SAURIA: TROPIDURIDAE) DA RESTINGA DE GURIRI, SÃO MATEUS, ES
}

\author{
TEIXEIRA, R. L. ${ }^{1}$ e GIOVANELLI, M. ${ }^{2}$ \\ ${ }^{1}$ Museu de Biologia Mello Leitão, Avenida José Ruschi, 4, CEP 29650-000, Santa Teresa, ES \\ ${ }^{2}$ Coordenação Universitária Norte do Espírito Santo (CEUNES), CEP 29930-000, São Mateus, ES \\ Correspondência para: Rogério L. Teixeira, Rua Bernardino Monteiro, 75, Centro, CEP 29650-000, \\ Santa Teresa, ES, Brasil, \\ Recebido em 17/04/98 - Aceito em 17/06/98 - Distribuído em 23/02/99
}

(Com 3 figuras)

\section{ABSTRACT \\ Ecology of Tropidurus torquatus (Sauria: Tropiduridae) of a sandy coastal plain of Guriri, São Mateus, ES, southeastern Brazil}

Ecological aspects of the collared lizard, Tropidurus torquatus, were studied monthly in a sandy coastal plain of southeastern Brazil, from February 1996 to March 1997. We collected 108 specimens using a pressure gun, of which 45 were males, 56 females, and 7 specimens which sex was not possible to identify. The snout vent length (SVL) ranged from 26.0 to $101.8 \mathrm{~mm}$ (mean $=61.7 \mathrm{~mm} ; \mathrm{SD}=15.1$ ). Total weight varied from 0.8 to $39.6 \mathrm{~g}$ (mean $=14.6 \mathrm{~g}$; SD =10.42). Based on the frequency of occurrence, major food items found in the stomach contents of T. torquatus were: ants (85.7\%), beetles (32.4\%), bees (31.4\%), termites (22.8\%), spiders (20.9) and flowers (20.0\%). Based on the relative number of prey ingested, main food items were: ants $(41.2 \%)$, and termites (40.8\%). Total number of prey found in the stomachs of the collared lizard was 2,903, which varied in individual stomachs from 1 to 268 (mean $=27.6 ; \mathrm{SD}=36.2$ ). Most of the time T. torquatus is a sit-and-wait predator. Its opportunistic feeding behavior, including invertebrates, small vertebrates (other lizard species), and vegetables (flowers and seeds), may contribute to the success of this lizard in colonizing a diversity of habitats.

Key words: Sauria, Tropiduridae, Tropidurus torquatus, coastal ecosystem, food habits, sex ratio, sandy coastal plain.

\section{RESUMO}

Aspectos da ecologia de Tropidurus torquatus foram estudados mensalmente na restinga de Guriri, São Mateus, Norte do Espírito Santo, de fevereiro de 1996 a março de 1997. Foram coletados 108 exemplares com arma de pressão, sendo 45 machos, 56 fêmeas e 7 exemplares cujo sexo não foi identificado. O comprimento rostro-anal no total dos exemplares variou de 26,0 a $101,8 \mathrm{~mm}$ (média $=$ $61,7 \mathrm{~mm}$; DP = 15,1). O peso no total dos exemplares variou de 0,8 a 39,6 g (média = 14,6 g; DP = 10,4). Em freqüência de ocorrência, os itens alimentares que predominaram na dieta de T. torquatus foram as formigas $(85,7 \%)$, coleópteros $(32,4 \%)$, abelhas $(31,4 \%)$, cupins $(22,8 \%)$, aranhas $(20,9)$ e flores $(20,0 \%)$. Em relação à frequiência numérica, os itens alimentares que predominaram foram as formigas $(41,2 \%)$ e cupins $(40,8 \%)$. O número total de presas encontrado nos conteúdos estomacais de T. torquatus foi de 2.903. Este número no total dos exemplares variou de 1 a 268 (média = 27,6; DP $=36,2$ ). Tropidurus torquatus é um predator do tipo senta-e-espera. O comportamento oportunístico desta espécie, cuja dieta inclui invertebrados, pequenos vertebrados (outros lagartos) e vegetais (flores e sementes), pode ser a explicação do sucesso deste lagarto em uma diversidade de hábitats.

Palavras-chave: Sauria, Tropiduridae, Tropidurus torquatus, ecossistema costeiro, alimentação, razão sexual, restinga. 


\section{INTRODUÇÃO}

Espécies do gênero Tropidurus ocorrem em áreas abertas na América do Sul e em Galápagos, sendo consideradas espécies onívoras com estratégias alimentares do tipo senta-e-espera (Araujo, 1987; Van Sluys, 1993, 1995). Apesar de sua ampla distribuição e diversidade (Rodrigues, 1987), informações sobre aspectos da auto-ecologia das espécies pertencentes ao gênero Tropidurus ainda são escassas e geralmente baseadas em poucos indivíduos (Rocha \& Bergallo, 1994).

Por ser uma espécie de lacertílio muito comum em áreas neotropicais, estudos envolvendo uma série de diferentes tópicos têm sido abordados recentemente. Aspectos taxonômicos do grupo $T$. torquatus têm sido amplamente verificado na literatura (Vanzolini et al., 1980; Rodrigues, 1987, 1988; Frost, 1992). Bergallo \& Rocha (1993, 1994) verificaram padrões de atividade, temperatura corporal e diferenciação espacial e alimentar entre T. torquatus e Cnemidophorus ocellifer. Divisão do espaço utilizado, assim como modificações morfológicas em T. torquatus foram avaliadas por Colli et al. (1992). Giaretta (1996) avaliou a dimensão do nicho utilizado por T. torquatus. Carpenter (1977) avaliou a coloração agressiva em três espécies pertencentes ao gênero Tropidurus da América do Sul. Vitt \& Goldberg (1983) estudaram a reprodução de $T$. torquatus. O hábito alimentar foi estudado por Bergallo \& Rocha (1994) e por Rocha \& Bergallo (1994).

O objetivo deste estudo foi evidenciar o papel ecológico que o lagarto Tropidurus torquatus desempenha na restinga de Guriri, localizada no Município de São Mateus, norte do Estado do Espírito Santo. Foi avaliada a dieta por sexo, número e tamanho de presas ingeridas, assim como alguns aspectos da reprodução.

\section{MATERIAL E MÉTODOS}

O trabalho de campo foi realizado em uma área de restinga da Praia de Guriri, município de São Mateus, Estado do Espírito Santo (ca. 18²5'S, 3944'W), de fevereiro de 1996 a março de 1997. O local possui relevo plano e é revestido por vegetação arbórea baixa, vegetação herbácea e gramíneas. O solo é constituído de depósitos arenosos aluviais de origem fluvio-marinhos (Suguio et al., 1982). O clima da região é quente e úmido (tipo
Aw de Köppen), com estação seca no outonoinverno e estação chuvosa na primavera-verão (Panoso et al., 1978). A temperatura média anual varia entre $22^{\circ} \mathrm{C}$ e $24^{\circ} \mathrm{C}$ e a precipitação média anual entre 1.000 e $1.250 \mathrm{~mm}$ (Nimer, 1989).

Os lagartos foram mortos por arma de pressão durante o período diurno (de 8:00 as 17:00 h). Os espécimens capturados foram fixados em formalina a $10 \%$, permanecendo uma semana nesta solução. Posteriormente, os exemplares foram lavados em água corrente e conservados em álcool a $70 \%$. Procedimentos adicionais de laboratório incluíram: medidas (comprimento rostro-anal em $\mathrm{mm}$, CRA), pesagens (balança digital, precisão $=0,1 \mathrm{~g}$ ) e dissecações para determinação do sexo e identificação do conteúdo estomacal. Foram quantificados os itens alimentares usando os métodos de freqüência de ocorrência e numérico. Presas encontradas inteiras foram contadas e medidas (comprimento total em $\mathrm{mm}$ ) com auxílio de paquímetro.

O comprimento e o peso médio entre machos e fêmeas foram comparados estatisticamente com análise de variância (ANOVA) com um critério. A relação entre número e o tamanho de presas intactas contra o comprimento rostro-anal de $T$. torquatus também foi avaliada. Diferenças na dieta entre sexos foram estatisticamente testadas usando o coeficiente de correlação de Spearman $\left(r_{s}\right)$. Abreviaturas usadas no texto foram: $\mathrm{DP}=$ desvio padrão; $r_{s}=$ coeficiente de correlação de Spearman; $\mathrm{GL}=$ grau de liberdade; $\mathrm{t}_{\mathrm{s}}=$ teste " $\mathrm{t}$ " para o coeficiente de correlação de Spearman. Espécimenstestemunho foram depositados na Coleção Zoológica do Museu de Biologia Mello Leitão (MBML).

\section{RESULTADOS}

\section{Hábitat e Hábitos}

Tropidurus torquatus é uma espécie comum na restinga de Guriri, que pode ser facilmente observada quando se caminha pela área. Os exemplares fogem à menor aproximação, mas podem ser observados sobre os terrenos dominados por areia, sobre troncos de pequenos arbustos inclinados e entre a densa concentração de cactos, comum na restinga estudada. Alguns espécimens vivem também nas proximidades das folhas do "coqueirinho de guriri” (Allagoptera arenaria, Arecaceae), cujas folhas pendentes ao chão fornecem excelentes abrigos para várias espécies de lagartos. Esporadicamente, 
podem ser vistos se deslocando no chão entre grupos de bromélias, as quais também fazem parte da vegetação da restinga de Guriri. Nos variados microhábitats, T. torquatus costuma esconder-se em pequenas tocas, onde provavelmente deve ficar durante as horas mais quentes do dia e durante a noite.

\section{Tamanho}

Foram capturados 108 exemplares de T. torquatus, sendo 45 machos, 56 fêmeas e 7 exemplares cujo sexo não foi identificado (Fig. 1). O comprimento rostro-anal no total dos exemplares variou de 26,0 a 101,8 mm (média = 61,7 mm; DP = 15,1). O comprimento rostro-anal nos machos variou de 32,1 a 94,0 mm (média $=67,5 \mathrm{~mm}$; DP $=16,1$ ), nas fêmeas de 33,2 a 101,8 mm (média $=60,0 \mathrm{~mm}$; $\mathrm{DP}=10,9)$, e nos nos exemplares cujo sexo não foi identificado de 26,0 a $54,0 \mathrm{~mm}$ (média $=37,3$ $\mathrm{mm} ; \mathrm{DP}=10,2)$. O tamanho médio dos exemplares diferiu significativamente entre machos e fêmeas (ANOVA: $\mathrm{F}_{1,99}=7,66 ; \mathrm{P}<0,0067$ ), evidenciando que, apesar do maior exemplar coletado ter sido uma fêmea, os machos geralmente são maiores.

O peso no total dos exemplares variou de 0,8 a 39,6 $\mathrm{g}$ (média $=14,6 \mathrm{~g}$; $\mathrm{DP}=10,4)$. O peso nos machos variou de 1,6 a 39,6 $\mathrm{g}$ (média $=20,2 \mathrm{~g}$; $\mathrm{DP}=12,8$ ), nas fêmeas de 1,8 a $28,8 \mathrm{~g}$ (média $=$ $11,5 \mathrm{~g}$; DP $=5,2)$, e nos juvenis de 0,8 a $7,7 \mathrm{~g}$ (média $=3,2 \mathrm{~g}$; DP $=2,6$ ). O peso também foi significativamente diferente entre sexos (ANOVA: $\mathrm{F}_{1,99}=$ 21,64; $\mathrm{P}<0,00017)$. Machos foram mais pesados que as fêmeas, apesar do fato de a maioria destas ter apresentado ovos ovidutais bem desenvolvidos.

\section{Dieta}

Dos 108 exemplares que tiveram os conteúdos estomacais examinados, apenas 3 estavam vazios. Tropidurus torquatus alimentou-se de 32 itens alimentares (Tabela 1), os quais variaram entre invertebrados, pequenos vertebrados e vegetais. Em termos de freqüência de ocorrência, os itens alimentares que predominaram na dieta de $T$. torquatus foram formigas $(85,7 \%)$, coleópteros $(32,4 \%)$, abelhas $(31,4 \%)$, cupins $(22,8 \%)$, aranhas $(20,9 \%)$ e flores $(20,0 \%)$. Numericamente, formigas $(41,2 \%)$ e cupins $(40,8 \%)$ foram os itens predominantes na dieta deste lagarto.

Foi feita uma comparação da dieta entre sexos (Tabela 2). Por freqüência de ocorrência, os principais itens alimentares predominantes na dieta dos machos foram formigas $(81,8 \%)$, seguidas por abelhas $(36,4 \%)$, flores $(31,8 \%)$ e cupins $(20,4 \%)$. Já na frequência numérica predominaram formigas (39,5\%), cupins $(37,4 \%)$ e abelhas $(13,7 \%)$. Demais categorias alimentares foram pouco relevantes na dieta dos machos. Com relação às fêmeas, os itens alimentares que predominaram na freqüência de ocorrência foram formigas $(90,9 \%)$, coleópteros $(38,2 \%)$, abelhas $(29,1 \%)$ e cupins $(25,4 \%)$. Em relação à frequência numérica foram formigas $(42,4 \%)$ e cupins $(41,6 \%)$. Não houve diferença significativa na dieta entre machos e fêmeas $\left(\mathrm{r}_{\mathrm{s}}=0,63 ; \mathrm{GL}=30 ; \mathrm{t}_{\mathrm{s}}=4,44 ; \mathrm{p}=0,0001\right)$.

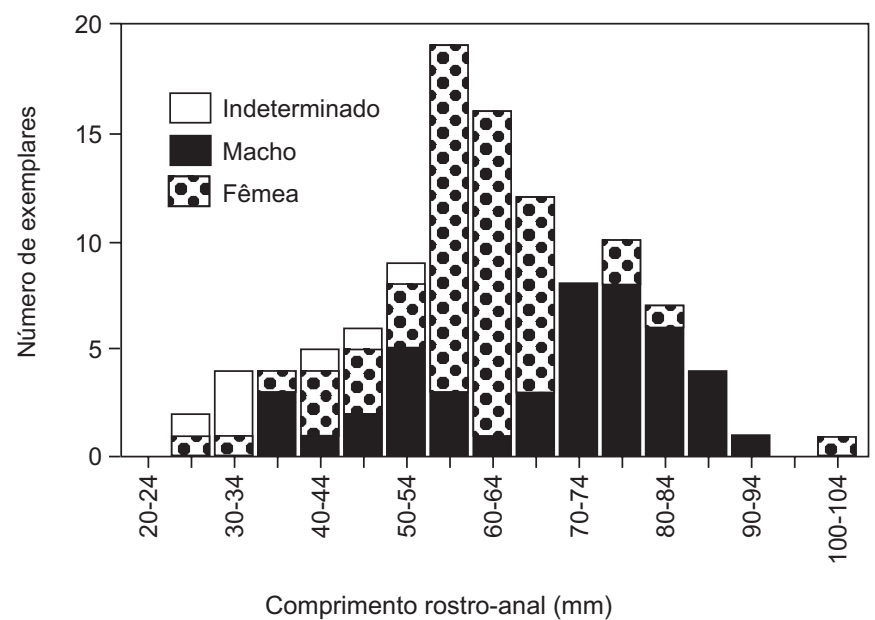

Fig. 1 - Distribuição de freqüências de comprimento em Tropidurus torquatus da restinga de Guriri, São Mateus, ES. 
TABELA 1

Itens alimentares encontrados nos conteúdos estomacais de Tropidurus torquatus da restinga de Guriri, São Mateus, ES. F.O. = freqüência de ocorrência; F.N. = freqüência numérica.

\begin{tabular}{l}
\hline Itens alimentares \\
\hline Orthoptera \\
Isoptera \\
Hemiptera (ninfa) \\
Hemiptera (adultos) \\
Coleoptera \\
Coleoptera (Elateridae) \\
Coleoptera (Scarabaeidae) \\
Coleoptera (Chrysomelidae) \\
Coleoptera (Dynastidae) \\
Coleoptera (larva) \\
Lepidoptera (larva) \\
Lepidoptera (adultos) \\
Homoptera \\
Neuroptera (larva) \\
Hymenoptera (Apidae) \\
Hymenoptera (Formicidae) \\
Blattariae \\
Odonata \\
Larvas insetos \\
Pupas de insetos \\
Restos de insetos \\
Isopoda \\
Araneae \\
Pseudoscopionida \\
Acarina \\
Chilopoda \\
Mabuya agilis \\
Gymnodactylus darwinii \\
Hemidactylus mabouia \\
Flores \\
Folhas \\
\hline
\end{tabular}

\begin{tabular}{|c|c|}
\hline F.O. (\%) & F.N. (\%) \\
\hline 11,4 & 0,5 \\
\hline 22,8 & 40,8 \\
\hline 1,9 & 0,1 \\
\hline 8,6 & 0,4 \\
\hline 32,4 & 2,2 \\
\hline 3,8 & 0,2 \\
\hline 2,8 & 0,2 \\
\hline 0,9 & 0,2 \\
\hline 1,9 & 0,2 \\
\hline 15,2 & 1,0 \\
\hline 6,7 & 0,3 \\
\hline 0,9 & 0,1 \\
\hline 3,8 & 0,2 \\
\hline 1,9 & 0,1 \\
\hline 31,4 & 8,0 \\
\hline 85,7 & 41,2 \\
\hline 8,6 & 3,4 \\
\hline 0,9 & 0,1 \\
\hline 5,7 & 0,2 \\
\hline 0,9 & 0,1 \\
\hline 6,6 & - \\
\hline 1,9 & 0,1 \\
\hline 20,9 & 1,0 \\
\hline 4,8 & 0,2 \\
\hline 0,9 & 0,1 \\
\hline 0,9 & 0,1 \\
\hline 2,8 & 0,1 \\
\hline 0,9 & 0,1 \\
\hline 2,8 & 0,1 \\
\hline 20,0 & 0,8 \\
\hline 0,9 & - \\
\hline 6,7 & 1,4 \\
\hline - & 2.903 \\
\hline
\end{tabular}

Não houve boa relação entre o número de presas ingeridas pelos indivíduos e o comprimento rostro-anal (Fig. 2). O número total de presas encontrados nos conteúdos estomacais de T. torquatus foi de 2903. Este número, no total dos exemplares, variou de 1 a 268 (média $=27,6$; DP = 36,2) (Fig. 2). O número de presas nos machos variou de 2 a 268 (média $=25,5 ; \mathrm{DP}=41,4)$, nas fêmeas de 1 a 149 (média $=29,5$; DP $=32,4$ ), e nos juvenis de 1 a 89 (média $=26,2$; DP $=32,9$ ). Números baixos de presas por estômago foram relacionados ao tamanho destas (por exemplo, lagartos), enquanto os números elevados foram associados a presas de pequeno porte (por exemplo, formigas, cupins etc). No que se refere ao tamanho das presas ingeridas, estas, geralmente, foram pequenas, não evidenciando nenhuma tendência à predação por presas maiores de acordo com o incremento de tamanho de T. torquatus (Fig. 3).

\section{Reprodução}

Fêmeas contendo ovos ovidutais foram encontradas ao longo de todos os meses amostrados. Este fato sugere uma desova prolongada para esta espécie estudada de lagarto da restinga. O número de ovos ovidutais encontrados nas fêmeas anali- 
sadas variou de 2 a 4 . Somente fêmeas maiores de $40 \mathrm{~mm}$ de comprimento rostro-anal apresentaram ovos ovidutais.

\section{DISCUSSÃO}

Tropidurus torquatus é a espécie de lagarto mais comum na restinga de Guriri. A plasticidade desta espécie no que se refere à ocupação de diferentes microhábitats e ao hábito alimentar oportunístico e generalista, confere fatores importantes que certamente contribuem para o sucesso da espécie na ocupação, adaptação, re- lação com outras espécies e a utilização dos recursos disponíveis dentro do espectro ambiental. Tropidurus torquatus co-ocorre com outras espécies de lacertílios na restinga de Guriri, sendo as seguintes observadas na área: Ameiva ameiva, Kenthropyx calcarata, Cnemidophorus ocellifer (Teiidae), Mabuya agilis, Mabuya sp (Scincidae), Hemydactilus mabouia e Gymnodactilus darwinii (Gekkonidae).

Moradores locais também informaram a presença do "teiú" (Tupinambis meriauae, Teiidae), mas nunca observamos este lagarto durante o trabalho de campo.

TABELA 2

Itens alimentares encontrados nos conteúdos estomacais de machos e fêmeas de Tropidurus torquatus da restinga de Guriri, São Mateus-ES. F.O. = freqüência de ocorrência; F.N. = freqüência numérica.

\begin{tabular}{l}
\hline Itens alimentares \\
\hline Orthoptera \\
Isoptera \\
Hemiptera (ninfa) \\
Hemiptera (adultos) \\
Coleoptera \\
Coleoptera (Elateridae) \\
Coleoptera (Scarabeidae) \\
Coleoptera (Crysomelidae) \\
Coleoptera (Dynastidae) \\
Coleoptera (larva) \\
Lepidoptera (larva) \\
Lepidoptera (adultos) \\
Homoptera \\
Neuroptera (larva) \\
Hymenoptera (Apidae) \\
Hymenoptera (Formicidae) \\
Blattariae \\
Odonata \\
Larvas de insetos \\
Pupa de insetos \\
Restos de insetos \\
Isopoda \\
Araneae \\
Pseudoscopionida \\
Acarina \\
Chilopoda \\
Mabuya agilis \\
Gymnodactylus darwinii \\
Hemidactylus mabouia \\
Flores \\
Folha \\
Frutos \\
Total de presas \\
\hline
\end{tabular}

\begin{tabular}{|c|c|}
\hline \multicolumn{2}{|c|}{ Machos } \\
\hline F.O. (\%) & F.N. (\%) \\
\hline 9,1 & 0,3 \\
\hline 20,4 & 37,4 \\
\hline 2,3 & 0,1 \\
\hline 4,5 & 0,1 \\
\hline 29,5 & 1,5 \\
\hline- & - \\
\hline 6,8 & 0,4 \\
\hline 2,3 & 0,5 \\
\hline 2,3 & 0,1 \\
\hline 11,4 & 0,8 \\
\hline 2,3 & 0,1 \\
\hline- & - \\
\hline 4,5 & 0,3 \\
\hline 4,5 & 0,2 \\
\hline 36,4 & 13,7 \\
\hline 81,8 & 39,5 \\
\hline 13,6 & 0,6 \\
\hline- & - \\
\hline 9,1 & 0,4 \\
\hline 2,3 & 0,1 \\
\hline 9,1 & - \\
\hline- & - \\
\hline 18,2 & 0,8 \\
\hline 9,1 & 0,4 \\
\hline- & - \\
\hline 2,3 & 0,1 \\
\hline 4,5 & 0,1 \\
\hline- & - \\
\hline 6,8 & 0,3 \\
\hline 31,8 & 1,4 \\
\hline- & - \\
\hline 6,8 & 0,4 \\
\hline
\end{tabular}

\begin{tabular}{|c|c|}
\hline \multicolumn{2}{|c|}{ Fêmeas } \\
\hline F.O. (\%) & F.N. (\%) \\
\hline 14,5 & 0,6 \\
\hline 25,4 & 41,6 \\
\hline 1,8 & 0,1 \\
\hline 12,7 & 0,6 \\
\hline 38,2 & 2,9 \\
\hline 5,4 & 0,2 \\
\hline - & - \\
\hline - & - \\
\hline 1,8 & 0,2 \\
\hline 18,2 & 1,1 \\
\hline 10,9 & 0,5 \\
\hline 1,8 & 0,1 \\
\hline 3,6 & 0,1 \\
\hline- & - \\
\hline 29,1 & 4,8 \\
\hline 90,9 & 42,4 \\
\hline 5,4 & 0,1 \\
\hline 1,8 & 0,1 \\
\hline 3,6 & 0,1 \\
\hline- & - \\
\hline 5,4 & - \\
\hline 3,6 & 0,1 \\
\hline 25,4 & 1,2 \\
\hline 1,8 & 0,1 \\
\hline 1,8 & 0,1 \\
\hline - & - \\
\hline 1,8 & 0,1 \\
\hline 1,8 & 0,1 \\
\hline- & - \\
\hline 12,7 & 0,5 \\
\hline 1,8 & - \\
\hline 7,3 & 2,3 \\
\hline & 1.624 \\
\hline
\end{tabular}




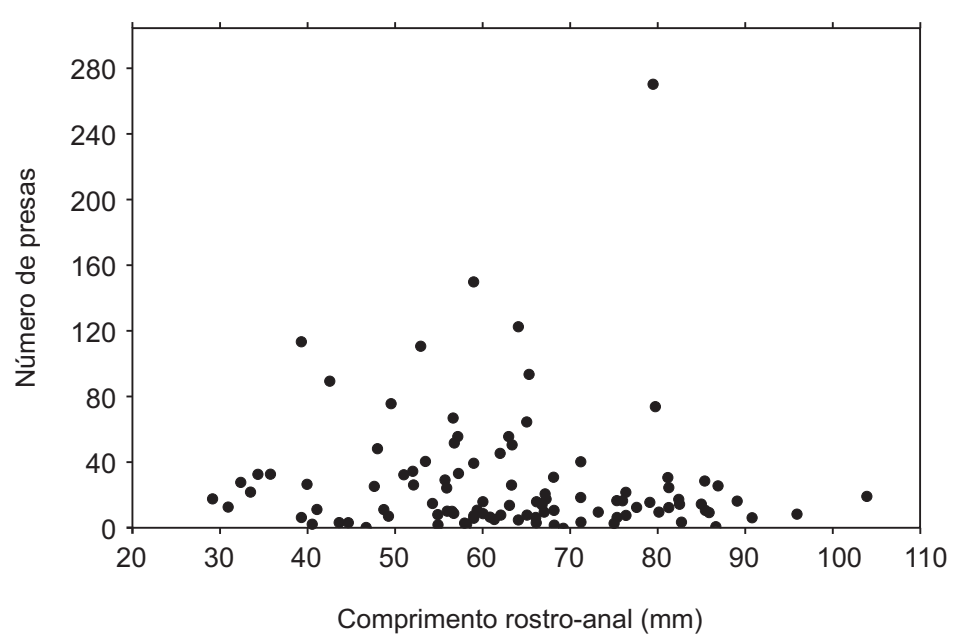

Fig. 2 - Relação entre o número de presas por estômago e o comprimento rostro-anal de Tropidurus torquatus da restinga de Guriri, São Mateus, ES.

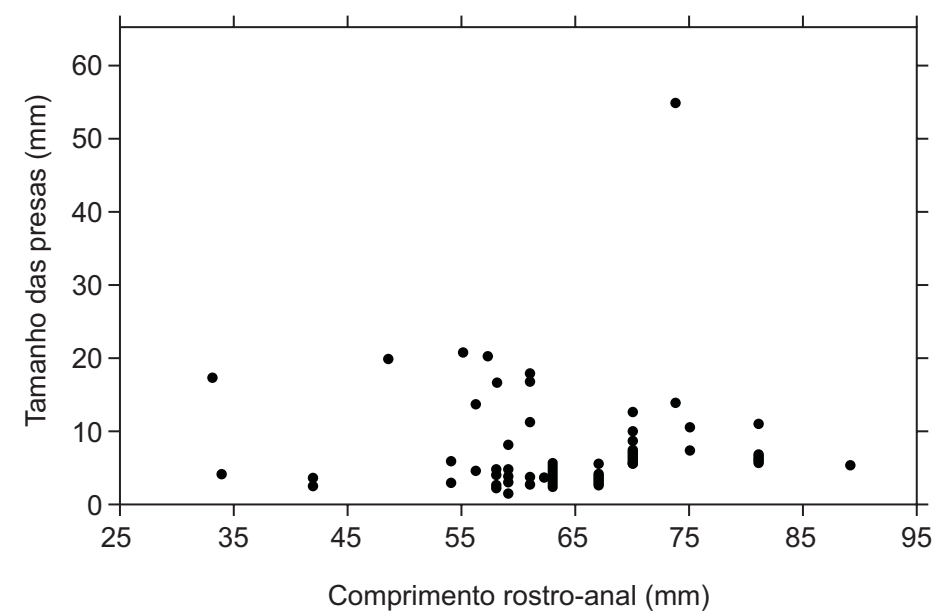

Fig. 3 - Relação entre o tamanho de presas intactas e o comprimento rostro-anal de Tropidurus torquatus da restinga de Guriri, São Mateus, ES.

Embora a estratégia de caça de T. torquatus tenha sido tradicionalmente adotada como do tipo senta-e-espera (Schoener, 1971), quando esta espécie ainda era incluída na família Iguanidae, as nossas observações evidenciaram mudanças de comportamentos no que se refere ao ataque às presas. Presas pequenas, tais como formigas e cupins, são predadas quase sem gasto de energia, uma vez que o predador geralmente ataca estes insetos sociais quando estão disponíveis em grande quantidades. No entanto, quando a espécie está à procura de abelhas, fica movendo-se de flor em flor, esperando o momento oportuno para investir contra as presas. Da mesma forma, pequenos lagartos podem não ter sido predados baseados apenas na tática de espreita. Araujo (1987) observou que Tropidurus de Carajás não é um típico senta-e-espera, patrulhando a área denominada de sítio de espera, podendo se deslocar rapidamente para a captura da presa dentro desta área. Além de variações no comportamento diário, é provável que estas variações ocorram também sazonalmente. Para Tropidurus itambere, por exemplo, foi observado diferenças no período de maior atividade entre inverno e verão, sendo esta espécie menos ativa durante os períodos de maior incidência solar (Van Sluys, 1992). 
Tropidurus torquatus da restinga de Guriri investe, principalmente, em pequenos artrópodos. Entre estes, formigas, cupins e abelhas contribuem com mais de $80,0 \%$ do total de presas encontradas no estômago. Rocha \& Bergallo (1994) avaliaram a dieta de $T$. torquatus de uma restinga próxima a nossa área de estudo. Estes autores observaram que os itens alimentares predominantes na dieta deste tropidurídeo foram as formigas, tanto numericamente $(46,1 \%)$, quanto volumetricamente (26,9\%). Cupins também foram importantes numericamente $(37,3 \%)$ e volumetricamente $(14,8 \%)$. Rocha \& Bergallo (1994) também constataram a presença de vegetais nos conteúdos estomacais, o que sugere ser sua ingestão comum e não acidental. Entretanto, flores poderiam ter sido ingeridas acidentalmente ao predarem abelhas, assim como pedaços de folhas ao predarem formigas em atividade de forrageamento. Ao predarem sobre sementes do "coqueirinho de guriri" (Allagoptera arenaria), T. torquatus pode também desempenhar o papel de disseminador da semente desta espécie de planta, uma das mais abundantes na área estudada.

A preferência por itens alimentares de pequeno tamanho, tais como formigas, cupins e abelhas, evidencia a principal estratégia alimentar da espécie. Se, por um lado, ao investir em pequenas presas a espécie economiza energia, a predação de poucos, porém grandes itens alimentares, tais como outros lacertílios, pode resultar numa economia de tempo e energia. É difícil avaliar qual estratégia poderia fornecer melhor aproveitamento energético, uma vez que predando pequenos artrópodos, $T$. torquatus têm necessariamente que ingeri-los em grande número. É provável que a espécie seja plástica suficiente para alternar sua tática de captura de acordo com a oferta de alimento, a qual deve se alterar ao longo do dia.

No que se refere à reprodução, T. torquatus aparentemente se reproduz durante todo o ano na restinga de Guriri, fato que pode ser especulado pela presença de fêmeas contendo ovos parcial e/ ou totalmente com casca ao longo dos meses. Isto pode ser um indicativo sobre o fato de ser a espécie de lacertílio mais comum na restinga estudada.

Agradecimentos - Ao CNPq pelo fornecimento de uma bolsa de recém-doutor (RLT, Proc. 302657/87-8), concedida ao primeiro autor. Hélio dos Santos Sá e Celso de Azevedo identificaram os insetos.

\section{REFERÊNCIAS BIBLIOGRÁFICAS}

ARAUJO, A. F. B.,1987, Comportamento alimentar dos lagartos: o caso do Tropidurus do grupo torquatus da Serra de Carajás, Pará (Sauria: Iguanidae). Anais de Etologia, 5: 189-197.

BERGALLO, H. G. \& ROCHA, C. F. D., 1993, Activity patterns and body temperatures of two sympatric lizards (Tropidurus torquatus and Cnemidophorus ocellifer) with different foraging tactics in south eastern Brazil. Amphibia-Reptilia, 14: 312-315.

BERGALLO, H. G. \& ROCHA, C. F. D., 1994, Spatial and trophic niche differentiation in two sympatric lizards (Tropidurus torquatus and Cnemidophorus ocellifer) with different foraging tactics. Austr. J. Ecol., 19: 17-25.

CARPENTER, C. C., 1977, The aggressive displays of three species of South American iguanid lizards of the genus Tropidurus. Herpetologica, 33(3): 285-289.

COLLI, G. R., ARAÚJO, A. F. B., SILVEIRA, R. \& ROMA, F., 1992, Niche partitioning and morphology of two syntopic Tropidurus (Sauria: Tropiduridae) in Mato Grosso, Brazil. J. Herpetol., 26(1): 66-69.

FROST, D. R., 1992, Phylogenetic analysis and taxonomy of the Tropidurus group of lizards (Iguania: Tropiduridae). Am. Mus. Novit., 3033: 1-68.

GIARETTA, A. A., 1996, Tropidurus torquatus. Home range. Herpetological Review, 27(2): 80-81.

NIMER, E., 1989, Climatologia do Brasil. IBGE, Rio de Janeiro, 422p.

PANOSO, L. A., GOMES, I. A., PIRES-FILHO, A. M. \& BONELLI, S., 1978, Levantamento de reconhecimento dos solos do Estado do Espírito Santo. Emprapa (Boletim Técnico, número 45), Rio de Janeiro, 461p.

ROCHA, C. F. D. \& BERGALLO, H. G., 1994, Tropidurus torquatus (Collared lizard). Diet. Herpetological Review, 25(2): 69 .

RODRIGUES, M. T., 1987, Sistemática, ecologia e zoogeografia dos Tropidurus do grupo torquatus ao sul do Rio Amazonas (Sauria, Iguanidae). Arq. Zool., São Paulo, 31: 105-230.

RODRIGUES, M. T., 1988, Distribution of lizards of the genus Tropidurus in Brazil (Sauria, Iguanidae). In: W. R. Heyer \& P. E. Vanzolini (eds.), Proceeding of a workshop on neotropical distribution patterns. Acad. Bras. Ciênc., Rio de Janeiro., pp. 305-315.

SCHOENER, T. W., 1971, Theory of feeding strategies. Ann. Rev. Ecol. Syst., 2: 369-404.

SUGUIO, K., MARTIN, L. \& DOMINGUEZ, J. M. L., 1982, Evolução da planície costeira do rio Doce (ES) durante o Quaternário: influência das flutuações do nível do mar. Atas do IV Simpósio do Quaternário no Brasil, pp. 93-116.

VAN SLUYS, M., 1992, Aspectos da ecologia do lagarto Tropidurus itambere (Tropiduridae), em uma área do sudeste do Brasil. Rev. Brasil. de Biol., 52(1): 181-185. 
VAN SLUYS, M., 1993, Food habits of the lizard Tropidurus itambere (Tropiduridae) in south eastern Brazil. $J$. Herpetol., 27(3): 347-351.

VAN SLUYS, M., 1995, Seasonal variation in prey choice by the lizard Tropidurus itambere (Tropiduridae) in southeastern Brazil. Ciência e Cultura, 47(1/2): 61-65.
VANZOLINI, P. E., RAMOS-COSTA, A. M. M. \& VITT, L., 1980, Répteis das Caatingas. Academia Brasileira de Ciências, Rio de Janeiro, 161p.

VITT, L. J. \& GOLDBERG, S. R., 1983, Reproductive ecology of two tropical iguanid lizards: Tropidurus torquatus and Platynotus semitaeniatus. Copeia, 1983(1): 131-141. 